\title{
3D RECONSTRUCTION OF WAVE-PROPAGATED POINT SOURCES FROM BOUNDARY MEASUREMENTS USING JOINT SPARSITY AND FINITE RATE OF INNOVATION
}

\author{
Zafer Dogan ${ }^{1,2}$, Ivana Jovanovic ${ }^{1,2}$, Thierry Blu ${ }^{3}$, and Dimitri Van De Ville , $^{1,2}$ \\ ${ }^{1}$ Medical Image Processing Lab (MIPLAB), Institute of Bioengineering, EPFL, CH-1015 Lausanne, Switzerland \\ ${ }^{2}$ Department of Radiology and Medical Informatics, University of Geneva, CH-1211 Geneva, Switzerland \\ ${ }^{3}$ Department of Electronic Engineering, Chinese University of Hong Kong, Shatin N.T. Hong Kong
}

\begin{abstract}
Reconstruction of point sources from boundary measurements is a challenging problem in many applications. Recently, we proposed a new sensing and non-iterative reconstruction scheme for systems governed by the three-dimensional wave equation. The points sources are described by their magnitudes and positions. The core of the method relies on the principles of finite-rate-of-innovation, and allows retrieving the parameters in the continuous domain without discretization.

Here we extend the method when the source configuration shows joint sparsity for different temporal frequencies; i.e., the sources have same positions for different frequencies, not necessarily the same magnitudes. We demonstrate that joint sparsity improves upon the robustness of the estimation results. In addition, we propose a modified multi-source version of Dijkstra's algorithm to recover the $Z$ parameters. We illustrate the feasibility of our method to reconstruct multiple sources in a 3-D spherical geometry.
\end{abstract}

Index Terms - Wave equation, finite rate of innovation, source localization, joint sparsity

\section{INTRODUCTION}

The linear inverse source problem has wide applications in biomedical imaging. Several mathematical models can apply, such as Poisson's equation for electroencephalography (EEG) [1], the heat equation for diffusive source localization [2], or the wave equation for acoustic sources [3]. Here, we focus on boundary measurements for systems goverend by the wave equation. The problem is illposed and thus challenging, and additional assumptions about the sources are required. Modern work has studied sparsity constraints, for example, compressed sensing approaches for radar imaging [4] or compact representations in adapted transforms $[5,6,7]$.

Here we exploit an explicit sparsity prior on the source model (i.e., a collection of point sources) and cast the reconstruction into the finite-rate-of-innovation (FRI) framework, as initially proposed in our preliminary work [3]. In the first step, we compute generalized samples to "sense" the sources using well-chosen test functions to relate the boundary measurements with useful volumetric information on the sources. Second, in the reconstruction step, we apply the FRI principle to these generalized samples to obtain the sources' locations, which is a non-linear estimation problem, by an equivalent root-finding problem.

This work was supported in part by the Swiss National Science Foundation (under the grants 2053530-132808 and PP00P2-123438) and in part by the Center for Biomedical Imaging (CIBM) of Geneva-Lausanne Universities and Hospitals, and the EPFL.
The contributions of this paper are twofold. First, we extend the method with a joint-sparsity constraint in order to improve the robustness of the estimation. Second, we propose to use a modified Dijkstra's shortest-path algorithm to solve for the axial $(Z)$ component of the source locations.

The paper is organized as follows. In Section 2, we introduce the problem formulation and the parameters to be estimated for the source recovery problem. In Sects. 3 and 4, we provide the details of the joint reconstruction algorithm. In Section 5, the reconstruction performance for single and joint annihilation algorithms is compared and we conclude in Section 6.

\section{PROBLEM FORMULATION}

Consider an unknown point source distribution within a volume $\Omega$ that creates a field according to the wave equation and measured on the boundary of a given volume, $\partial \Omega$. Observing the time harmonic solutions of the wave equation of the form $u(\mathbf{r}, t)=e^{i \omega t} U(\mathbf{r})$ and $p(\mathbf{r}, t)=e^{j \omega t} P(\mathbf{r})$, we obtain the inhomogeneous Helmholtz equation

$$
\nabla^{2} U+k^{2} U=-P,
$$

where $U$ and $P$ are the field and the source terms, respectively, and $k^{2}=\frac{\omega^{2}}{c^{2}}$ is the wave number with $\omega$ being the angular frequency of the wave.

In order to well-pose the source recovery problem [8], we further parametrize the source distribution such that

$$
P(r)=\sum_{m=1}^{M} c_{m} \delta\left(\mathbf{r}-\mathbf{r}_{\mathbf{m}}\right),
$$

where $c_{m} \in \mathbb{R}, M \in \mathbb{Z}^{+}$are the magnitudes, and $r_{m} \in \Omega$ the locations of the sources. We restate the problem as reconstruction of source positions and magnitudes knowing the pressure field only on the boundary by $\left.U\right|_{\partial \Omega}$ and $\left.\nabla U\right|_{\partial \Omega}$ for some finite number of temporal frequencies.

\section{SENSING AND ANNIHILATION}

We provide the generalized sensing method to extract the volumetric source information from the boundary field measurements and develop a non-iterative joint-reconstruction algorithm based on joint sparsity and finite rate of innovation.

\subsection{Generalized Sensing}

We propose a sensing method to retrieve the samples of the volumetric source distribution, termed generalized samples, from the 
boundary measurements of the generated wave field with the carefully selected sensing functions.

According to the second Green's identity, the generalized samples of the unknown source distribution can be obtained by

$$
\langle\Psi, P\rangle=\oint_{\partial \Omega}(\Psi \nabla U-U \nabla \Psi) \cdot \overline{\partial S}
$$

where $\overline{\partial S}$ is in the outward surface normal direction, provided that the sensing function satisfies

$$
\nabla^{2} \Psi=-k^{2} \Psi \text { within } \Omega .
$$

The proposed family of sensing functions satisfying (4) are characterized by

$$
\Psi_{l}[n]=\frac{e^{i k_{l} z}}{x+i y-a_{n}}, \quad a_{n}=a e^{i n \alpha}, a_{n} \notin \Omega,
$$

where $l$ represents the chosen frequency with the corresponding wavenumber $k_{l}$, and $a_{n}$ 's are the poles of the sampling function located on the XY-plane with equidistant radial angles, $\alpha$, outside the domain $\Omega$. This choice of the sensing functions allows us to robustly reconstruct the projections of the locations of the point sources on the XY-plane by annihilating the generalized samples. For the jointsparse source model, we simply extract generalized samples using the same family of test functions, but at different frequencies.

\subsection{Joint Annihilation}

The generalized samples extracted according to (3) satisfy the equality

$$
\mu_{l}[n]=\left\langle\Psi_{l}[n], P\right\rangle=\sum_{m=1}^{M} \frac{c_{m} e^{i k_{l} z_{m}}}{s_{m}-a_{n}}=\frac{\sum_{m=0}^{M-1} c_{m}^{\prime} e^{i m n \alpha}}{\prod_{m=1}^{M}\left(s_{m}-a_{n}\right)}
$$

for the selected wavenumber $k_{l}, l \in \llbracket 1, L \rrbracket$ and $n \in \llbracket 1, N \rrbracket$, and $s_{m}=x_{m}+i y_{m}$. Hence, it is clear that the exponential terms in the numerator of (6) can be annihilated by an FIR digital filter, $h$, characterized by

$$
H(z)=\prod_{r=0}^{M-1}\left(1-e^{i r \alpha} z^{-1}\right)=\sum_{r=0}^{M} h_{r} z^{-r} .
$$

Defining a polynomial for the denominator of the (6) as $X\left(a_{n}\right)=$ $\prod_{m=1}^{M}\left(s_{m}-a_{n}\right)=\sum_{q=0}^{M} x_{q} a_{n}^{q}$ with $x_{M}=1$, the annihilation equation has the following form

$$
\left\{\mu_{l}[\cdot] X(a .)\right\} * h=0, \text { for all } l \in \llbracket 1, L \rrbracket .
$$

In terms of matrix representation, (8) can be formulated as:

$$
\mathbf{A}_{l} \mathbf{x}=\mathbf{H D}_{l} \mathbf{V x}=\mathbf{0},
$$

where $\mathbf{H}$ is an $(N-M) \times N$ Toeplitz matrix representing the annihilating filter $h, \mathbf{D}_{l}$ is an $N \times N$ diagonal matrix of generalized samples, $\mathbf{V}$ is an $N \times(M+1)$ Vandermonde matrix of poles of the sensing function; i.e., $a_{n}$ and $\mathrm{x}$ is the unknown vector of $M+1$ polynomial coefficients with $x_{M}=1$. For the noiseless case, the system $\mathbf{A}_{l} \mathbf{x}=\mathbf{0}$ has $(N-M)$ equations with $M$ unknowns. Hence, we conclude that for this setup we need at least $N=2 M$ generalized samples.
For joint reconstruction of the source locations, we extend the above linear system of equations so as to incorporate multiple generalized samples taken at different frequencies, i.e., from the following set of linear subsystems

$$
\mathbf{H D}_{l} \mathbf{V x}=0
$$

$$
\mathbf{H D}_{L} \mathbf{V x}=0 \text {, }
$$

we construct a large system of the form

$$
\mathbf{A x}=\mathbf{B C V x}=0,
$$

where $\mathbf{B}$ is an $L(N-M) \times L N$ block diagonal matrix of $\mathbf{H}$ matrices, $\mathbf{C}$ is an $L N \times N$ matrix formed by vertically concatenating $\mathbf{D}_{l}$ matrices. From (11), we conclude that it is possible to jointly reconstruct the point source locations.

\subsection{Model Mismatch and Denoising}

The linear system $\mathbf{A x}=\mathbf{0}$ is solved in the least-squares sense to extract the source locations on XY-plane. For the noiseless case, the method achieves exact solution, but in practice the measurements are corrupted with noise. Hence, one needs to compensate for the measurement noise model mismatches.

Assuming that the generalized samples are corrupted with complex AWGN; i.e., $\hat{\mu}_{l}[n]=\mu_{l}[n]+v[n]$ where $v$ denotes the complex noise, we propose to denoise the generalized samples with the following Cadzow-like denoising scheme [9].

As mentioned earlier, the subsystem matrix, $\mathbf{A}_{l}=\mathbf{H D}_{l} \mathbf{V}$, is of rank $M$ in the noiseless case. In the proposed denoising scheme, we exploit this fact to denoise generalized samples extracted for different frequencies separately. In order to allow noise and model mismatch, we first assume higher number of sources $\tilde{M}>M$ to construct $\tilde{\mathbf{H}}$ and $\tilde{\mathbf{V}}$. We then obtain the corresponding unitary matrices, i.e., $\tilde{\mathbf{H}}_{0}$ and $\tilde{\mathbf{V}}_{0}$ to precondition the system matrix such that $\tilde{\mathbf{A}}_{l 0}=\tilde{\mathbf{H}}_{0} \hat{\mathbf{D}}_{l} \tilde{\mathbf{V}}_{0}$.

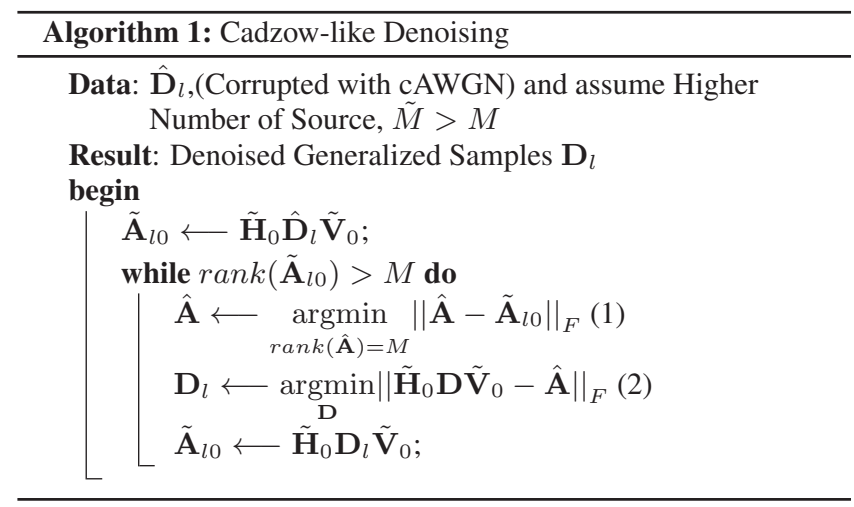

We propose two-step iterations for the Cadzow-like denoising scheme. First, we find the low-rank approximation of $\tilde{A}_{l 0}$ minimizing an objective (1) in Algorithm 1 by forcing the last $\tilde{M}-M$ singular values of it to be zero. Second, we extract the denoised generalized samples as a minimization of the objective (2) in Algorithm 1 . We continue the iterations until the $(M+1)^{t h}$ largest singular value of $\tilde{\mathbf{A}}_{l 0}$ is smaller than a predefined threshold. 


\section{MAGNITUDE AND Z-AXIS ESTIMATION}

\subsection{Magnitude Recovery}

In order to completely describe the source distribution, one still has to determine the z-positions of the source locations and the magnitudes. The estimation of these parameters are done with the same set of generalized samples. Considering (6) with the XY-locations estimated in the previous step we obtain another linear system

$$
\mu_{l}[n]=\sum_{m=1}^{M} \frac{c_{m} e^{i k_{l} z_{m}}}{s_{m}-a_{n}}, n \in \llbracket 1, N \rrbracket,
$$

where $\mu_{l}[n]$ are the denoised generalized samples and $s_{m}=x_{m}+$ $i+y_{m}$ are the estimated XY-plane positions. In matrix notation, (12) can be represented as $\mathbf{E f}_{l}=\mu_{l}^{T}$ with the following explicit representations:

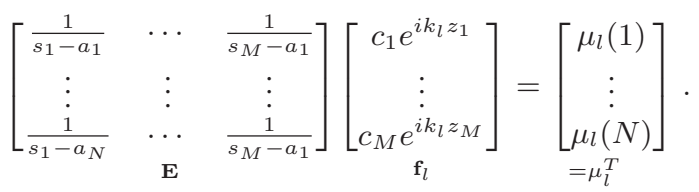

The solution to this system is given by $\mathbf{f}_{l}=\mathbf{E}^{\dagger} \mu_{l}^{T}$, where ${ }^{\dagger}$ represents the pseudoinverse of a matrix. Here, it is possible to find a frequency-variant magnitude solution depending on the problem formulation. Assuming that the magnitudes are also jointly sparse the joint reconstruction of the magnitude of $L$ set of generalized samples will be the mean of the magnitude solution sets:

$$
\mathbf{C}=\left[\begin{array}{c}
c_{1} \\
\vdots \\
c_{M}
\end{array}\right]=\frac{1}{L} \sum_{l=1}^{L}\left|\mathbf{f}_{l}\right|
$$

\subsection{Periodicity Cancellation for the Z-Solutions}

For the $Z$ component of the source locations, the problem is harder than that of the magnitude. The main difficulty is that the phase of $P_{l}$ is periodic. Therefore, for each $z_{m}$, we have a set of periodic solutions within the domain of $\Omega$ as follows:

$$
\mathbf{Z}(l)=\left[\begin{array}{c}
z_{1}(l) \\
\vdots \\
z_{M}(l)
\end{array}\right]=\frac{\arg \left(\mathbf{f}_{l}\right)+2 \pi n_{l}}{k_{l}}, \forall\left\{\begin{array}{l}
n_{l} \in \mathbb{Z} \\
z_{m}(l) \in \Omega \\
l=1: L
\end{array} .\right.
$$

We note that if the common period of $L$ solutions lies outside of the domain $\Omega$, then there is a unique solution which has the same value among all possible solution sets from $\mathbf{Z}(1)$ to $\mathbf{Z}(L)$ for the noiseless case. Therefore, we propose a solution for the z-location as a modified multi-source Dijkstra's algorithm for closed paths as the minimization of the following objective:

$$
\begin{aligned}
& \hat{n}_{l}=\underset{l}{\operatorname{argmin}} \sum_{l=2}^{L}\left|\frac{\arg \left(\mathbf{f}_{1}(m)\right)+2 \pi n_{1}}{k_{1}}-\frac{\arg \left(\mathbf{f}_{l}(m)\right)+2 \pi n_{l}}{k_{l}}\right| \\
& \mathbf{Z}=\left[\begin{array}{c}
z_{1} \\
\vdots \\
z_{M}
\end{array}\right]=\frac{1}{L} \sum_{l=1}^{L} \frac{\arg \left(\mathbf{f}_{l}\right)+2 \pi \hat{n}_{l}}{k_{l}}
\end{aligned}
$$

We assume that the selected frequencies are sorted in increasing order such that the set $z_{m}(1)$ is the smallest set among other $L-1$ possible solution sets and set as the initial reference set $\mathrm{R}$. At each iteration, we compare the reference set (R) with a search set (S), which is the solution to the next higher frequency. We chose a set $\mathrm{N} \subset \mathrm{S}$ and $\mathrm{N} \equiv \mathrm{R}$ such that each element in $\mathrm{R}$ has its closest equivalent in set $\mathrm{N}$. Then the set $\mathrm{D}$ keeps the distance between the two closest sets and set $\mathrm{T}$ keeps the corresponding solutions. Once we select the solutions sets among all frequencies we choose mean of the set having the shortest path as our solution. The proposed method is summarized in Algorithm 2.

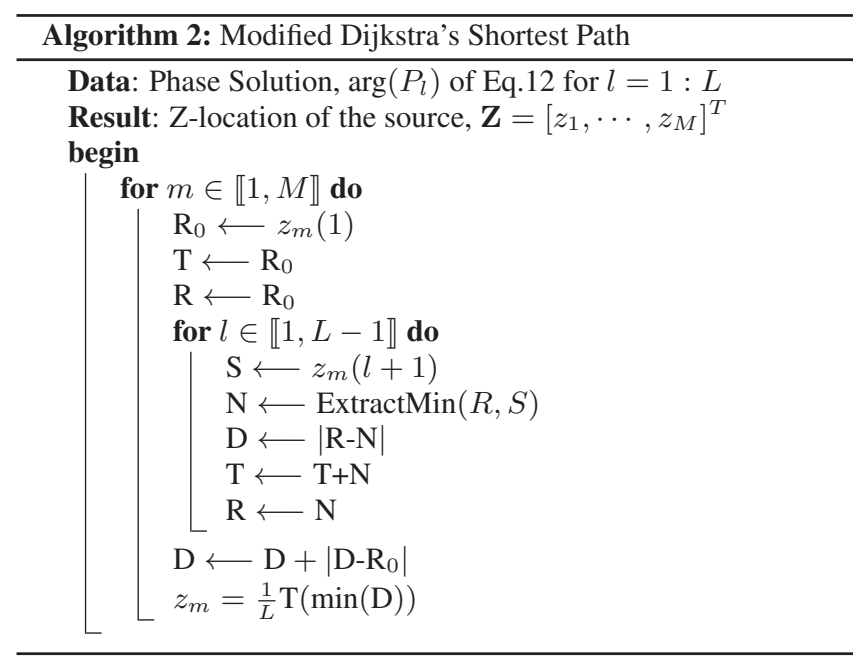

\section{EXPERIMENTAL RESULTS}

We perform numerical experiments to asses the performance of our new reconstruction algorithm for a variety of source configuration by observing the localization quality of joint reconstruction with respect to varying number of frequencies. Our settings are inspired by from photoacoustic imaging [10], which is an imaging modality with promising biomedical applications including early detection of breast cancer and small animal imaging.

For the experimental setup, we consider a spherical volume of radius $1 \mathrm{~cm}$ where the source points are generated randomly. Moreover, we assume that the generalized samples are available from the field measurements on the boundary of the volume of interest with a frequency range of $1 \mathrm{MHz}$ to $1.2 \mathrm{MHz}$ with a $50 \mathrm{kHz}$ separation $\left(f=f_{0}+(0\right.$ to $L) \times \Delta f$ with $f_{0}=1 \mathrm{MHz}$ and $\Delta f$ is $\left.50 \mathrm{kHz}\right)$ which is reasonable in the photoacoustic applications. Here the separation step-size is chosen such that the beating frequency between the distinct frequencies guarantees a unique solution within the domain $\Omega$. Once we have the generalized samples, we further degrade them with cAWGN to evaluate the performance of the localization and denoising algorithms.

In the first experiment, we compare the observed estimation variance of the parameters with respect to the theoretical limits given by CRLBs for a single acoustic source. In Fig. 1 (a),(b) and (d), we compare the performance of the estimations along $\mathrm{X}$ and $\mathrm{Y}$ axes and the magnitude for single and multiple frequency reconstruction, respectively. As expected, the joint-reconstruction method performs better as we have more frequency measurements. For the Z-localization, as the joint-reconstruction algorithm needs at least two different set of measurements to choose the proper period of the solution, we only compare for multiple frequencies in Fig. 1 (c). Here, it is clear that by adding more jointly sparse generalized samples, the algorithm can reach to CRLB for a lower SNR level. 
Table 1: RMSE $\left(10^{-4}\right)$ per source for 100 realizations of randomly generated sources using generalized samples (20dB). $L$ indicates the number of frequencies.

\begin{tabular}{l|c|c|c|c|c|c|c}
\hline \hline & \multicolumn{3}{|c|}{ number of sources } & \multicolumn{3}{c}{ number of sources } \\
\hline$L$ & 1 & 3 & 5 & $L$ & 1 & 3 & 5 \\
\hline 2 & 13.1 & 13.2 & 13.6 & 4 & 9.4 & 11.5 & 13.1 \\
\hline 3 & 11.0 & 12.3 & 13.2 & 5 & $\mathbf{6 . 4}$ & $\mathbf{9 . 3}$ & $\mathbf{1 2 . 1}$ \\
\hline \hline
\end{tabular}

For the second experiment, we consider only the localization error for multiple source estimation problem in terms of RMSE per source. From Table 1, we see that the average reconstruction error can be decreased by jointly reconstructing more frequencies. This result is especially interesting to control the error per source in localizing more source points in the model. Finally, we provide a sample scatter plot for localization of three sources with two and five frequencies in Fig. 2. We observe the XY-plane estimations on Figs. 2 (a) and (c) and XZ-plane estimations on Figs. 2 (b) and (d) where we clearly observe the periodicity behavior in estimation along the Z-axis.

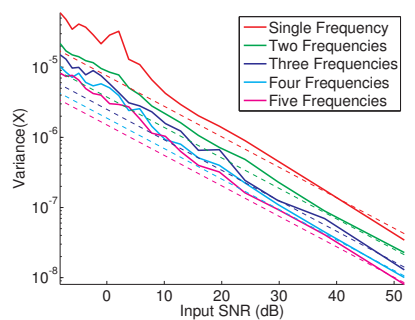

(a)

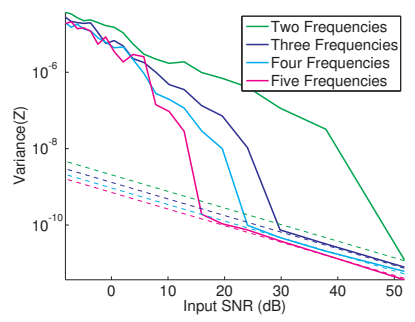

(c)

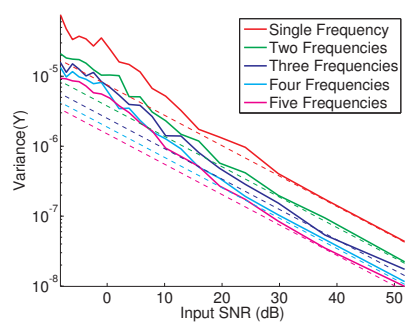

(b)

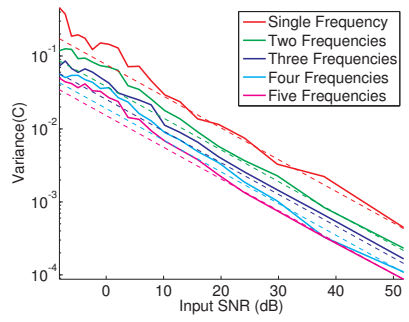

(d)
Fig. 1: Comparison of CRLBs for a single monopole source configuraiton (dashed line) with observed variance (solid line) for 100 realizations and different number of frequencies. (a) Variance of estimated X locations. (b) Variance of estimated Y locations. (c) Variance of estimated Z locations. (d) Variance of estimated magnitude.

\section{CONCLUSIONS}

We investigated the joint-sparsity source model for the localization of point sources from boundary measurements of a 3D wave field. We proposed an extension to our previous sensing and reconstruction algorithm incorporating multiple measurements of the field at different frequencies. Comparison between the estimation performance and the theoretical limits, revealed that the proposed jointsparse sensing and reconstruction algorithm achieves the CRLBs for lower SNR levels than the previous algorithm which only considers single frequency measurement.

The results for multiple source localization showed that jointsparsity source models can be very useful in robust estimation of the parameters for low SNR regime. We believe that our method has a promising application in improving the detection of multiple source points in generation photo/thermo acoustic imaging.

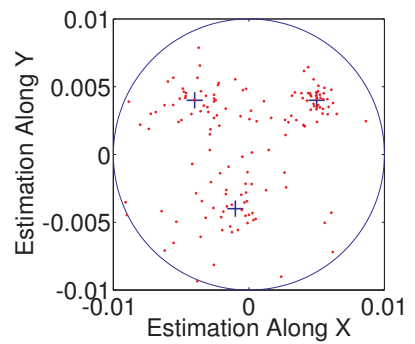

(a)

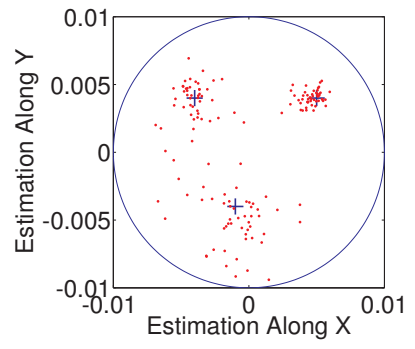

(c)

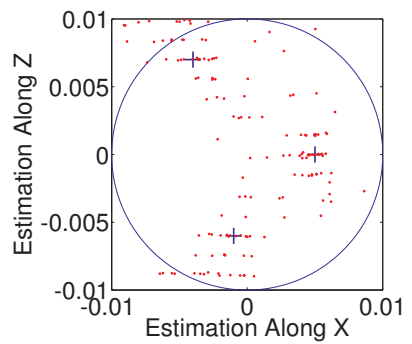

(b)

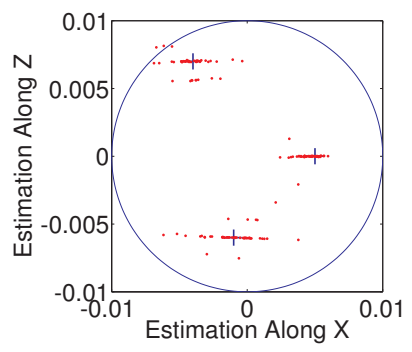

(d)
Fig. 2: Scatter plot of estimated locations for 50 realizations using generalized samples (20dB) at multiple frequencies: $L=2$ (top); $L=5$ (bottom). The (+) indicate the ground truth and (.) the estimated locations

Sparse source models for the localization of source distributions from overdetermined boundary field measurements remains as a promising research area of further research. Moreover, we also consider the possibility and feasibility of the proposed method for photoacoustic imaging applications.

\section{REFERENCES}

[1] D. Kandaswamy, T. Blu, L. Spinelli, C. Michel, and D. Van De Ville, "Local mulilayer analytic sensing for EEG source localization: Performance bounds and experimental results," in Proceedings of the Fifth IEEE International Symposium on Biomedical Imaging: From Nano to Macro (ISBI'11), March 30-April 2, 2011, pp. 479-483.

[2] I. Jovanovic, L. Sbaiz, and M. Vetterli, "Tomographic approach for parametric estimation of local diffusive sources and application to heat diffusion," in Image Processing, 2007. ICIP 2007. IEEE International Conference on, 16 2007-oct. 19 2007, vol. 4, pp. IV -153 -IV -156.

[3] Z. Dogan, V. Tsiminaki, I. Jovanovic, T. Blu, and D. Van De Ville, "Localization of point sources for systems governed by the wave equation," 2011, vol. 8138, p. 81380P, SPIE.

[4] Albert Fannjiang, Penchong Yan, and Thomas Strohmer, "Compressed remote sensing of sparse objects," SIAM J. Imag. Sci., vol. 3, pp. 596618, 2010.

[5] E. Candes and L. Demanet, "The curvelet representation of wave propagators is optimally sparse," Comm. Pure Appl. Math.

[6] Michael Liebling and Michael Unser, "Autofocus for digital fresnel holograms by use of a fresnelet-sparsity criterion," J. Opt. Soc. Am. A, vol. 21, no. 12, pp. 2424-2430, Dec 2004.

[7] M. Nikolic, Gongguo Tang, and A. Nehorai, "3d electromagnetic imaging using compressive sensing," in Sensor Array and Multichannel Signal Processing Workshop (SAM), 2010 IEEE, oct. 2010, pp. 37 -40.

[8] V Isakov, "Uniqueness and stability in multi-dimensional inverse problems," Inverse Problems, vol. 9, no. 6, pp. 579, 1993.

[9] J. A. Cadzow, "Signal enhancement: A composite property mapping algorithm," IEEE Trans. on Acoust., Speech, Signal Processing, vol. 36, pp. 39-62, 1988.

[10] Habib Ammari, Emmanuel Bossy, Vincent Jugnon, and Hyeonbae Kang, "Mathematical modeling in photoacoustic imaging of small absorbers," SIAM Rev., vol. 52, pp. 677-695, November 2010. 